\title{
THEORETICAL ASSESSMENT OF TECHNOLOGICAL POTENTIAL OF MAGNETIC AND ELECTRIGAL SEPARATION
}

\author{
V.V. KARMAZIN \\ Moscow State Mining University, Prospekt Lenina 6, \\ 117935 Moscow, Russia \\ (Received January 22, 1996, in final form February 18,1997)
}

\begin{abstract}
$\underline{\text { Abstract }}$
Magnetic, electrical an combined methods of mineral beneficiation are widely used in various branches of mining industry. These processes have significant economic and ecological advantages in those areas where they can be applied technologically. It is thus necessary to analyse technological possibilities and areas of potential applications. Different designs of the separators must also be considered. Such an attempt is being done in this article based on the assessment of the level of different types of electromagnetic forces and their role in the force regimes of mineral separation. Fundamental directions and limitations of increasing the efficiency of forces of the electromagnetic origin used for mineral separation. Equations for the calculation of these forces are also given. Contribution of the magnetic moment of the particles (magnetoadhesive forces) is also included.
\end{abstract}

\section{INTRODUCTION}

Methods of magnetic, electrical and combined separation possess high technical and economic efficiency and where their technological application is justified, these techniques are exceptionally competitive. One of the most important advantages of ore beneficiation in magnetic and electrical fields is that these fields can be superimposed on other physical fields according to the superposition principle, so that these fields do not distort the existing fields. Combined force modes of 
separation can thus be created employing several properties of minerals simultaneously.

Another important advantage of these processes is a weak dependence on medium in which the separation is carried out which allows them to be easily combined with other processes in various process flowsheets. High level of separation forces in magnetic, electrical and combined fields allows not only to increase the recovery of finely ground mineral particles but also to ensure increased throughput. In view of a rather low cost of separators this is of a significant technico-economic advantage.

Combination of high-intensity force regimes (compactness, high throughput and efficiency of magnetic separators) with environmental friendliness makes magnetic, electrical and combined techniques preferable, particularly for underground mining and beneficiation operations, for mobile and modular plants as well as for regions with limited supply of water (dry separation).

Intensive development, in the last few years, of mineral separation processes in magnetic and electric fields has been induced not only by their technico-economical advantages, but also by deterioration of the ore upgradeability as a result of reduction of the quality of ores, in terms of composition and particle size.

Under these conditions, new processes extend lifetime of the traditional technological approaches by allowing to recover metals from slimes and low-grade middlings by selective removal of slimes and other variants of elimination of the inefficiencies in the existing solutions.

As has been mentioned above, beneficiation of mineral resources in magnetic and electric fields has been, during the recent years, one of the fastest developing techniques. New direct, inverse and combined processes of minerals beneficiation., shown in Fig. 1, have been developed 


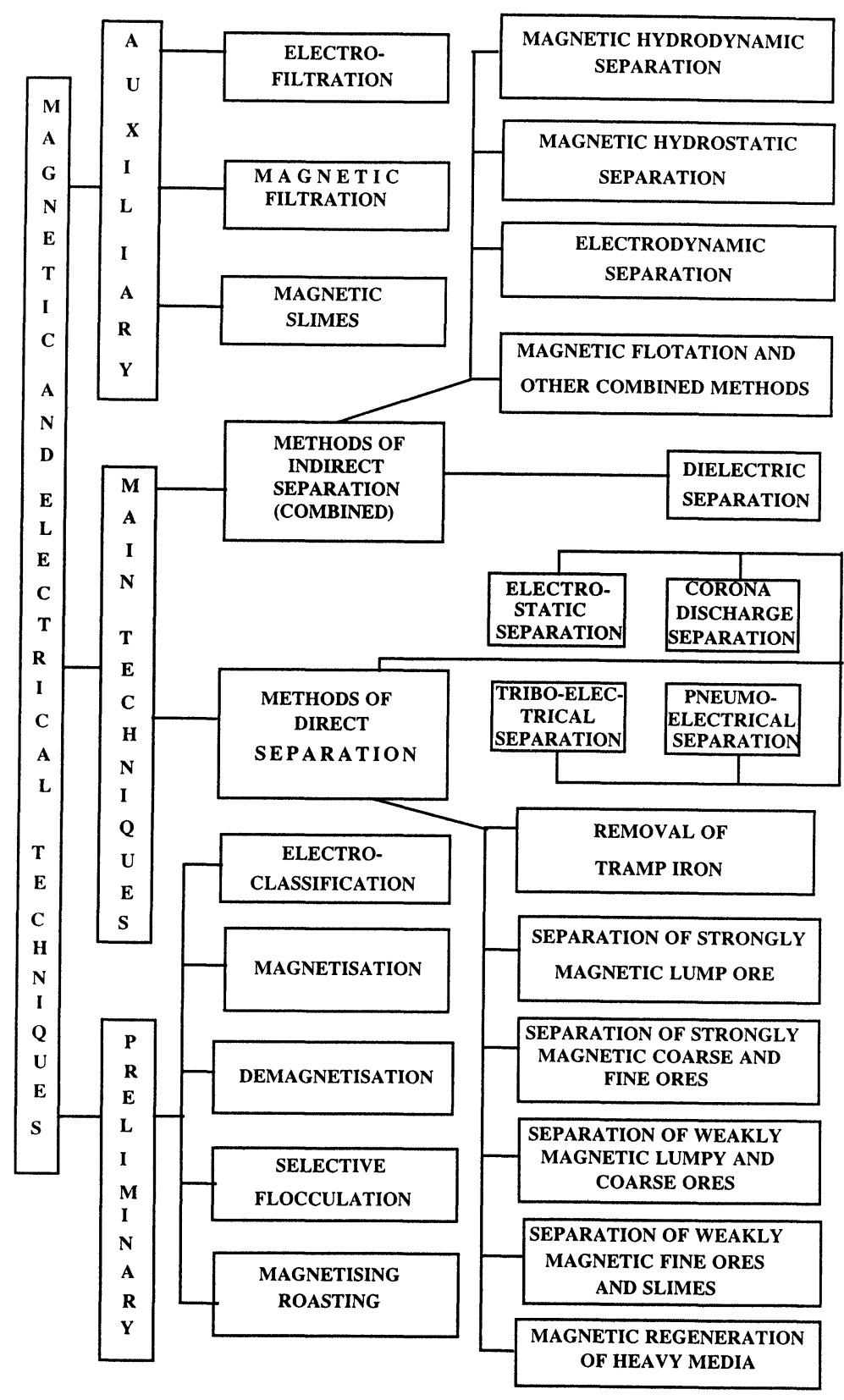

Fig. 1 Classification of the magnetic and electrical methods of concentration 


\section{NEW SEPARATION PROCESSES IN ELECTROMAGNETIC FIELDS}

New processes of beneficiation employ not only electromagnetic properties of minerals, but also fully independent physical and/or physico-chemical properties. These are, for instance, density, particle size and shape, surface properties, floatability etc. Typically, these new processes include high-gradient magnetic separation, magneto-adhesive separation, volume-gradient separation, ferrohydrostatic, magnetohydrostatic and electrodynamic separations, separation with magnetic carriers, flotation in magnetic fields, separation in a magnetite suspension, dynamic and thermomagnetic separation, magnetic concentration on inclined planes and other processes. Technical possibility of magnetic separation of ions also looks promising.

Currently, of particular importance appears to be high-gradient separation in strong magnetic fields. In essence, it is a magneto-hydromechanical process in which a large part of the working space is controlled by hydromechanical forces.

Reduction of the capture zone, or localisation of forces in the vicinity of capture surfaces, while certain conditions are met, leads to a sharp increase of the ponderomotive forces. However, kinetic optimisation of mass transfer in the working space becomes necessary. It is necessary to ensure maximum probability of particles entering the capture zone, in the process of mass transfer during separation. It was proved experimentally more than twenty tears ago [1].

Over the recent years, this process has been introduced in Russia as well as abroad. It is being used in order to improve existing flowsheets of the beneficiation plants or to introduce new technical and technological solutions. High-gradient magnetic separation also allowed to re-open previously closed nonviable mining operations. In Russia, HGMS became essential for the upgrading of the oxidised iron ores and of ores of rare metals. Taking into account that all sulphur-containing minerals in coal possess magnetic properties, it becomes apparent that introduction of HGMS into processes of coal beneficiation is a realistic way of increasing the efficiency of coal desulphurisation at lower cost compared with existing techniques. This is a direct way of protection of environment against acid rain. 
For the first time, superconducting separators were investigated in Russia (Moscow Mining Institute and the Baikov Institute of Metallurgy) with our participation in 1960s [2]. A wide spectrum of technical problems of application of magnetic, electrical and combined processes of separation can be easily solved using strong-current high-temperature superconductors. Although this approach will be viable only in the future, individual operations can be efficiently realised using superconducting magnetic systems operating at liquid helium temperatures.

Advantages of large volumes of magnetic field with high intensity available in superconducting magnetic systems not always exceed drawbacks associated with a difficulty of obtaining high gradients of the magnetic field. Therefore, principles of increasing ponderomotive forces in the working volume of electromagnetic separators requires thorough investigation. This is the way of increasing the force regime of separation and thus of technological potential of separators.

The situation can be illustrated by the following example. High-gradient magnetic separation using superconducting magnetic systems allows to recover weakly magnetic mineral complexes with particle size down to $1 \mu \mathrm{m}$. On the other hand, such a separation is not possible using similar or even stronger superconducting systems with open gradient (i.e. low gradient without ferromagnetic matrix).

Advantages of superconducting magnetic systems can be clearly seen in a combined process of magnetohydrodynamic separation where the recovering force is directly proportional to the current density and magnetic induction in the working space. That is why by increasing the magnetic induction as a result of the superconducting system the current density in electrolyte can be reduced. The most serious technical obstacle for creating of a MHD-separator is removed [1].

\section{FUNDAMENTAL TYPES OF THE SEPARATING FORCES}

All ponderomotive forces generated in electric and magnetic fields exhibit themselves either in alternating fields (Lorentz or Ampere forces), or in static fields (Coulomb force). In the latter case, the forces can be divided into two subgroups, namely electrostatic and magnetostatic forces (Fig. 2). Therefore, three 


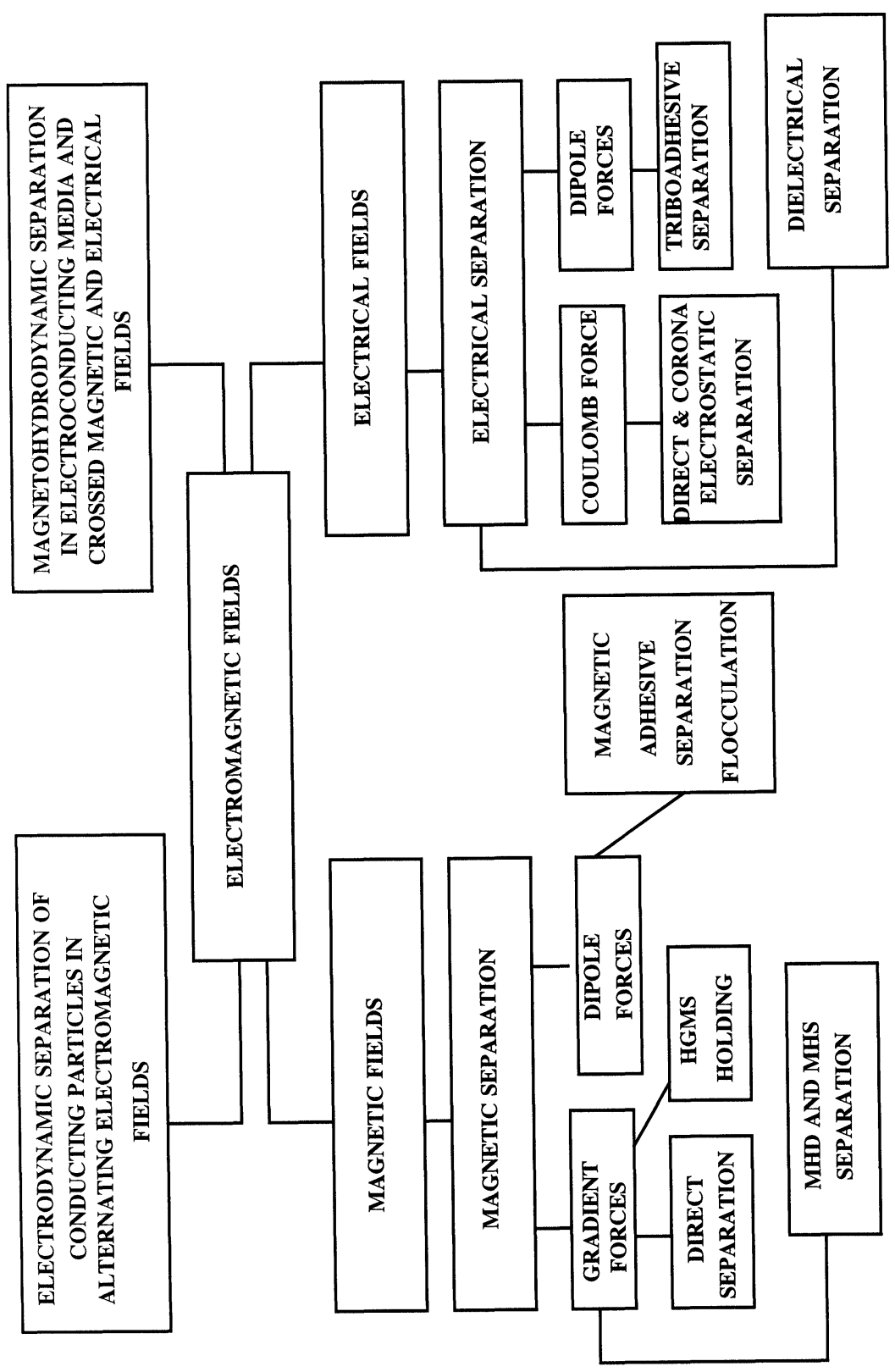

Fig. 2 Ponderomotive electromagnetic forces used in the processes of separation of minerals 
types of forces are used: electromagnetic (magnetohydrodynamic and electrodynamic separators), magnetic (direct and reverse separation, high-gradient and magnetoadhesive separation, magnetic flocculation etc.), and electrical (direct and reverse separation, triboadhesive separation etc.).

Strictly speaking, term magnetostatic force is conditional, and is not correct on molecular level. On macroscopic level, e.g. for mineral crystals and their composition it is, however, fully acceptable.

It is well known that, based on its physical nature, all variations of ponderomotive magnetic forces, are associated with Lorentz interaction of the magnetic field with electrically charged particles moving in this field, or with electromagnetic interaction of moving charges (current) through the magnetic field generated by these charges [3].

On macroscopic level, it is possible to identify those processes comprising electrical and magnetic fields, including the running fields. and in various environments, also processes existing in stationary fields based on intrinsic magnetic and electric charges of particles.

Direct processes include interaction of particles, having a magnetic moment, with the field as a result of ponderomotive forces in a neutral medium. Reverse processes can occur only in such a medium that possesses either magnetic or electric permeability. This permeability must be intermediate compared with permeabilities of the minerals to be separated. Particles with smaller permeability will be expelled by the medium, since the magnetic force $\left(M_{\text {mag }}-M_{\text {medium }}\right) \nabla H$ changes its direction ("minus" sign).

Reverse processes with magnetic or electrical expulsion of particles from the field (magnetohydrostatic or dielectric separation) have exactly the same relationship to gravitational beneficiation as direct processes of magnetic or electrical separation. Both processes can occur in absence of the gravity force $\left(^{*}\right)$, i.e. they cannot be called gravitational.

$\left.{ }^{*}\right)$ This is clearly incorrect. In the absence of the gravity force the gravitational constant is zero and the apparent density of a magnetic fluid approaches infinity and the process of magnetohydrostatic separation ceases to exist. (Editor's comment) 
Processes that use the image forces, forces of dipolar interaction, and particularly high-gradient magnetic separation deserve particular attention. We shall thus dwell on some theoretical assessments of a possibility to intensify the force regime of separation of minerals as a consequence of these forces.

An elementary magnetic moment (e.g. atomic), placed in a non-homogeneous magnetic field, is acted upon by a force couple (a torque trying to align the vector of magnetic moment $\boldsymbol{M}$ along the direction of the external magnetic field $\boldsymbol{H}_{\mathrm{e}}$ ):

$$
\mathbf{T}=-\left[\mathbf{M} \cdot \mathbf{H}_{\mathrm{e}}\right]
$$

At the same time, a ponderomotive force is acting on the magnetic moment:

$$
\mathbf{F}_{\mathrm{m}}=-\nabla \mathrm{U}=-\nabla\left(\mathbf{M} \cdot \mathbf{H}_{\mathrm{e}}\right)
$$

where $\mathrm{U}=\left(\mathbf{M} \cdot \mathbf{H}_{\mathrm{e}}\right)$ is the potential energy of the magnetic dipole in the external magnetic field $H_{\mathrm{e}}$.

Exact derivation of these formulae can be found in well-known publications [3] which allows us to accept these relationships as a basis of analysis of the effect of the ponderomotive force in the working space of a magnetic separator.

In a process of magnetic beneficiation, particles which can be conditionally considered to be magnetic dipoles, are suspended in a flow of water (or air). As a rule, their concentration does not prevent them from rotating unhindered. Thus, independently from conditions and principles of summation of atomic magnetic moments inside a particle, the particle aligns itself rapidly in such a way that the effective magnetic moment $M$ of every particle becomes parallel to the direction of the external magnetic field $H_{\mathrm{e}}$.

As a result of this fact the force couple can be neglected when evaluating the ponderomotive force (but not when assessing tension of the flocs). It is, however, always necessary to remember the orientational effect induced bu the force couple.

The traction component of the ponderomotive magnetic force, in accordance with its great role in magnetic separation, is of considerably greater interest to us than 
the torque, although it is somewhat more difficult to be assessed. Using operations of vector analysis and dropping the minus sign indicating the direction of the force based on decrease of the potential energy, eq.(2) can re-written as:

$\mathbf{F}_{\mathrm{m}}=\nabla\left(\mathbf{M} \cdot \mathbf{H}_{\mathrm{e}}\right)=\left(\mathbf{H}_{\mathrm{e}} \nabla\right) \mathbf{M}+\mathbf{M}\left(\nabla \mathbf{H}_{\mathrm{e}}\right)+\left[\mathbf{H}_{\mathrm{e}} \operatorname{curl} \mathbf{M}\right]+\left[\mathbf{M} \operatorname{curl} \mathbf{H}_{\mathrm{e}}\right]$

As a result of properties of the magnetic moment, the first and the third terms on the right-hand side of eq. (3) are equal to zero and thus we can write:

$$
\mathbf{F}_{\mathrm{m}}=\nabla\left(\mathbf{M} \cdot \mathbf{H}_{\mathrm{e}}\right)=(\mathbf{M} \cdot \nabla) \mathbf{H}_{\mathrm{e}}+\left[\mathbf{M} \cdot \operatorname{curl} \mathbf{H}_{\mathrm{e}}\right]
$$

In that part of the working space of a separator where free electric currents are absent we have $\operatorname{curl} \mathrm{H}_{\mathrm{e}}=0$ and eq. (4) can be rewritten:

$$
\mathbf{F}_{\mathrm{m}}=\mathbf{M} . \nabla \mathbf{H}_{\mathrm{e}}=\frac{\mathrm{M}_{\mathrm{x}} \partial \mathrm{H}_{\mathrm{e}}}{\partial \mathrm{x}}+\frac{\mathrm{M}_{\mathrm{y}} \partial \mathrm{H}_{\mathrm{e}}}{\partial \mathrm{y}}+\frac{\mathrm{M}_{\mathrm{z}} \partial \mathrm{H}_{\mathrm{e}}}{\partial \mathrm{z}}
$$

As we have seen above, in magnetic separation a vector $M$ of every magnetisable particle in a suspension aligns parallel the intensity of the external magnetic field $\mathbf{H}_{\mathrm{e}}$ and the vector of force $\mathbf{F}_{\mathrm{m}}$ must have a direction of the gradient of the magnetic field. Taking this into account, eq. (5) can be written as:

$$
\mathbf{F}_{\mathrm{m}}=\mathbf{M} \cdot \nabla \mathrm{H}=\mathbf{M} \operatorname{grad} \mathrm{H}_{\mathbf{e}}
$$

We have to point out, however, that the condition curl $\mathbf{H}_{\mathrm{e}}=0$ accepted above, ceases to be valid when magnetic particles make contact among themselves or with a ferromagnetic pole. This is the result of the fact that magnetic moments of the particles, their molecular currents and transfer currents become parts of direct interaction. In such a case real magnitude of their mutual attraction can be considerably greater than the calculated value.

Generally speaking, accuracy of formulae for calculation of ponderomotive force, for different boundary conditions, depends only on how accurately is distribution of elementary magnetic moments is taken into account, and how accurately is their summation carried out within the volume of a magnetisable particle. In a limiting case it is possible to simplify this complicated procedure. For instance, in [3], based on an assumption that the mean magnetic moment of a magnetisable 
particle is constant in its entire volume, this moment is expressed through the effective magnetisation (magnetic susceptibility and magnetic field intensity). Such an assumption is permissible only for very small values of atomic magnetic moments and when the exchange interaction is absent. This occurs only for diamagnetic and weakly magnetic materials.

We can write:

$$
\mathbf{M}=\mathbf{J} \mathbf{V}=\kappa \mathbf{H} \mathbf{V}
$$

where $J$ is the magnetisation, $V$ is the volume of a particle and $\kappa$ is the magnetic susceptibility of a particle. Introducing (7) into (4), and ignoring the difference between external field $H_{\mathrm{e}}$ and internal field $H_{\mathrm{i}}$ in a magnetic material, we formally obtain:

$$
\mathbf{F}_{\mathrm{m}}=\kappa \mathrm{V}(\mathbf{H} \nabla \mathbf{H}+[\mathbf{H} \text { curl } \mathbf{H}])=0.5 \kappa \mathrm{VVH}^{2}=\kappa \mathrm{VHVH}
$$

Equation (8) is till today being used in research reports on magnetic beneficiation as a fundamental and practically the only expression for the calculation of the magnetic force. This formula is being used not only in those cases where it is permissible, but also in those cases where it is, from first principles, not permissible.

For transition from the field of elementary magnets $H_{\text {micro }}$ to the field of mineral particles $H_{\text {macro }}$, a single average of the magnetic field intensity within a magnetic particle is not sufficient, even when taking into account a contribution of the system of elementary magnetic moments. Using a method of superposition of external and internal fields in a magnetic material, it is necessary to consider, apart from external fields in a magnetic material, also a supplementary field of an assembly of elementary magnets (close neighbouring atoms), although of a form of a demagnetising field.

A more accurate expression for (8) can be obtained by substituting (7) into (6) and by taking into account differences between $\mathbf{H}_{\mathrm{e}}$ and $\mathbf{H}_{\mathrm{i}}$ :

$$
\mathbf{F}_{\mathrm{m}}=\kappa \mathrm{VH}_{\mathrm{igradH}}=\chi \mathrm{mH}_{\mathrm{i}} \operatorname{gradH}_{\mathrm{e}}
$$


where $\chi=\kappa / \rho$ is the mass magnetic susceptibility and $m$ is the mass.

It is well known that for spherical magnetisable particles

$$
\mathrm{H}_{\mathrm{i}}=\frac{3 \mathrm{H}_{\mathrm{e}}}{\mu+2}
$$

and the difference between external and internal fields becomes important for magnetic permeability $\mu>1$. Even more complicated is the calculation of the ponderomotive magnetic force for ferromagnetic particles for which not only is $\mu>$ 1 , but also their magnetic susceptibility and permeability are complex functions of the intensity of the magnetising field.

In this case, and particularly because of non-homogeneity of the magnetising field $H_{\mathrm{e}}=\mathrm{f}(\mathrm{x}, \mathrm{y}, \mathrm{z})$, the susceptibility and permeability must be considered as non-linear tensors $\left\|\kappa\left(\mathrm{H}_{\mathrm{e}}\right)\right\|$ and $\left\|\mu\left(\mathrm{H}_{\mathrm{e}}\right)\right\|$. These tensors depend on $H_{\mathrm{e}}$ which, in turn, is a function of coordinates. The application of tensor analysis does not improve clarity in the calculation of the magnetic force. Although the index (tensor) form of representation of such quantities is more descriptive than the symbolic (vector) representation, the calculation is, in frequent cases, possible only by numerical methods.

From the point of view of our objective to increase the ponderomotive force, equation (9) gives an answer to a question about realistic limits of the magnetic field strength for beneficiation of weakly magnetic slimes. In fact, $\mathbf{H}_{\mathbf{i}}$ increases the magnetisation of a particles only up to the level of saturation $\mathbf{J}_{\mathbf{s}}$, i.e. $\lim \kappa \mathrm{H}_{\mathbf{i}}=\mathrm{J}_{\mathbf{s}}$, for $\mathrm{H} \rightarrow \infty, \kappa \rightarrow 0$. In such a case, $F_{\mathrm{m}}=\mathrm{VJ}_{\mathrm{s}} \operatorname{gradH}_{\mathrm{e}}$. Therefore, the magnetic force increases with $H_{\mathrm{e}}$ only when gradH $\mathrm{e}_{\mathrm{e}}\left(\nabla \mathrm{H}_{\mathrm{e}}\right)$ increases.

Magnetic saturation of strongly magnetic minerals (ferromagnetic, antiferromagnetic) is usually achieved in the interval of $H_{\mathrm{e}}$ between 10 to $20 \mathrm{kOe}$. Material of pole pieces is usually a mild steel or ARMCO, with $B_{\mathrm{S}}=20$ to $25 \mathrm{kG}$ and therefore an increase of the magnetic field strength up to these limits also increases magnetic moment and field gradient in the vicinity of the pole tips:

$$
\mathbf{B}=\mathbf{H}_{\mathrm{e}}+\left.4 \pi \mathbf{J}\right|_{\mathrm{B} \rightarrow \mathrm{B}_{\mathbf{s}}}=\mathbf{H}_{\mathrm{e}}+4 \pi \mathbf{J}_{\mathrm{s}}
$$


When $H_{\mathrm{e}}$ is increased above the level of saturation of metal, the overall induction keeps growing as a result of $H_{\mathrm{e}}$. However, magnetic moment of a particle, gradient of the field and, therefore, magnetic force, do not increase.

Thus, if the upper limit of the magnetic field strength in the magnetohydrodynamic separation is determined only by the cost and possibilities of the magnetic system, then for direct separation this upper limit has a physical meaning, and a further increase in the magnetic field intensity, without an increase in the field gradient, is not worthwhile.

\section{THE ROLE OF THE LOCAL MAGNETIC FIELD OF PARTICLES}

It is necessary to keep in mind that a particle itself, as a magnetic dipole, creates around itself a high gradient of the magnetic field and can attach itself even to a smooth surface of a pole with a high magnetic permeability with the force of "mirror image":

$$
\mathbf{F}_{\text {magn }}=\mathbf{M} \frac{\mathrm{dH}_{\mathrm{d}}}{\mathrm{d} \mathbf{x}}=\mathbf{M} \frac{\mathrm{d}\left(\frac{\mathbf{M}}{\mathbf{x}^{3}}-\right)}{\mathrm{d} \mathbf{x}}=\frac{3 \mathbf{M}^{3}}{\mathrm{x}^{4}}
$$

where $H_{\mathrm{d}}$ is the magnetic field intensity of a particle dipole and $x$ is the distance of the particle from pole surface.

This magnetoadhesive force, of a large value for immediate contact between small particles and the pole, decreases to zero when the magnetisation of the pole material approaches saturation. In such a case, full magnetic saturation reduces the ponderomotive force. However, it is necessary to keep in mind that such a saturation depends on the shape of the poles and occurs at much higher values of $H_{\mathrm{e}}$ compared to those that can be found in tables and that refer to a torroidal shape.

Equation (11) allows to explain the results of an experiment shown in Figure 3. This experiments allows to determine disagreement between experimental data and calculations using eq. (8) (curve 1 in Figure 4). The curve 2 corresponds to 


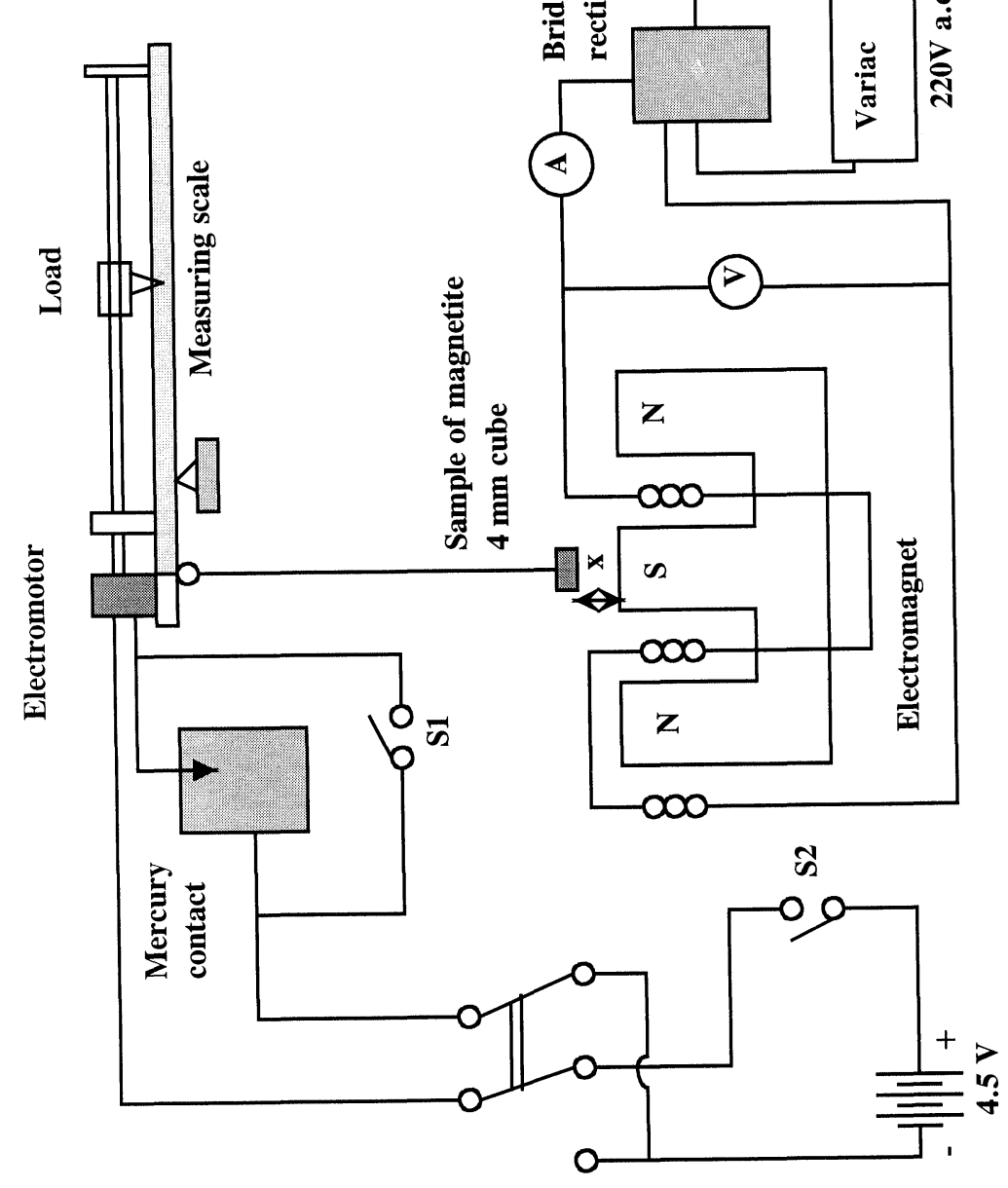

Fig. 3 Schematic diagram of an experimental set-up used to measure the dependence of the magnetic force on the width of a gap $x$

magnetoadhesive forces of the dipole interaction (mirror image) which can be calculated using eq. (11) for 1st and 2nd Gaussian positions, as shown in Figure 5. 


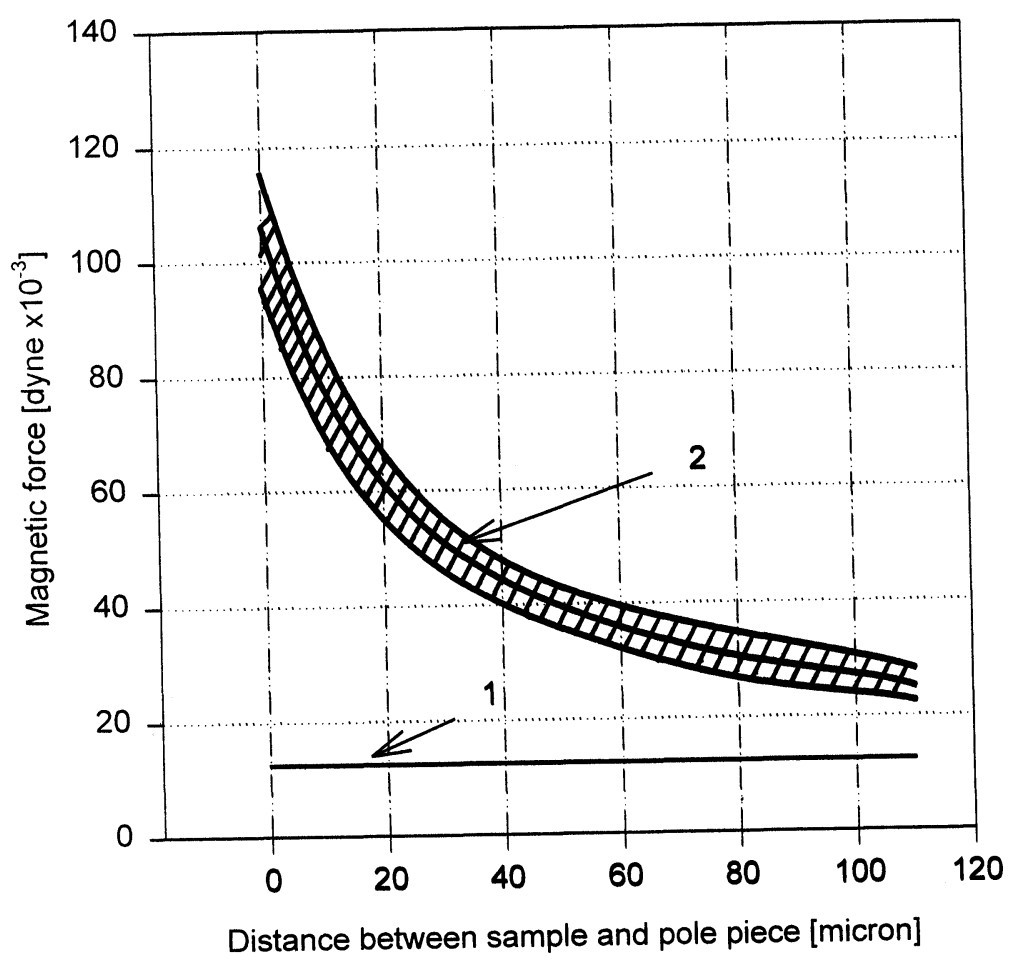

Fig. 4 Magnetic force as a function of the distance between a sample and the pole piece. Curve 1 was calculated according to eq. (8), while curve 2 was determined experimentally.

It was established in this experiment that the real level of ponderomotive magnetic forces within the area of contact of a particle with a pole exceeds, for one or two orders of magnitude, the force calculated from eq. (8). This can be explained only by the presence of the additional and essential contribution of the force of the mirror image of the dipole interaction of a particle with the dipole [4].

The elementary act of flocculation, namely agglomeration of two magnetisable particles also occurs as a result of the traction dipole interaction between the particles, caused by the symmetrical non-uniformity of the total field of two particles, or more accurately, by field gradients shown in Figure 6. We have 


\section{1st Gaussian Position}

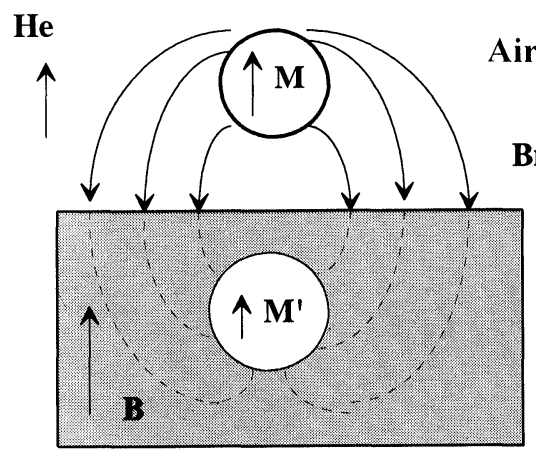

ir (2)

$M^{\prime}=M \frac{\mu_{1}-1}{\mu_{1}+1}$

$\mathrm{Bn} 1=\mathrm{Bn} 2$

$\mu_{1} \gg 1$

$B_{1} \approx H_{e}$

2nd Gaussian Position

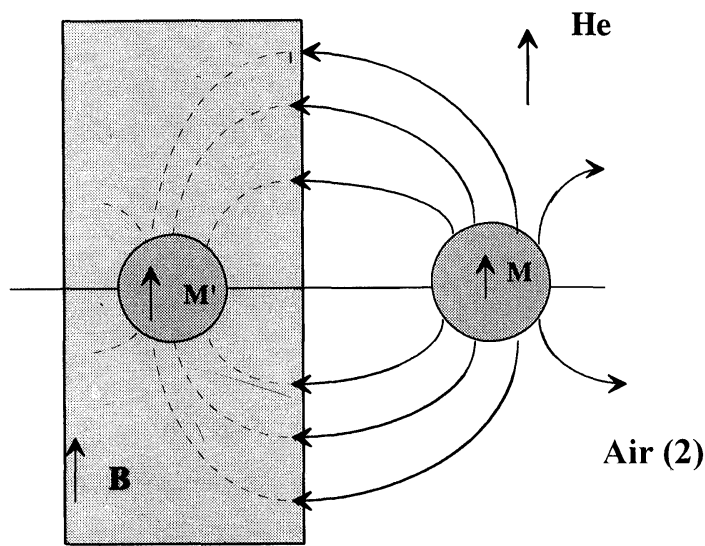

$$
M^{\prime}=M \frac{\mu_{1}-1}{\mu_{1}+1}
$$

$\mu_{1} \gg 1$

$B>>H_{e}$

Ferromagnetic material (1) $\quad \frac{B_{t 1}}{B_{t 2}}=\frac{\mu_{1}}{\mu_{2}}$

Fig. 5 Schematic diagram of the mirror image forces generated between a uniformly magnetised sphere and the surface of a ferromagnetic pole. Magnetic field in the vicinity of the pole is homogeneous (grad $\left.\mathrm{H}_{\mathrm{e}}=0\right)$. 

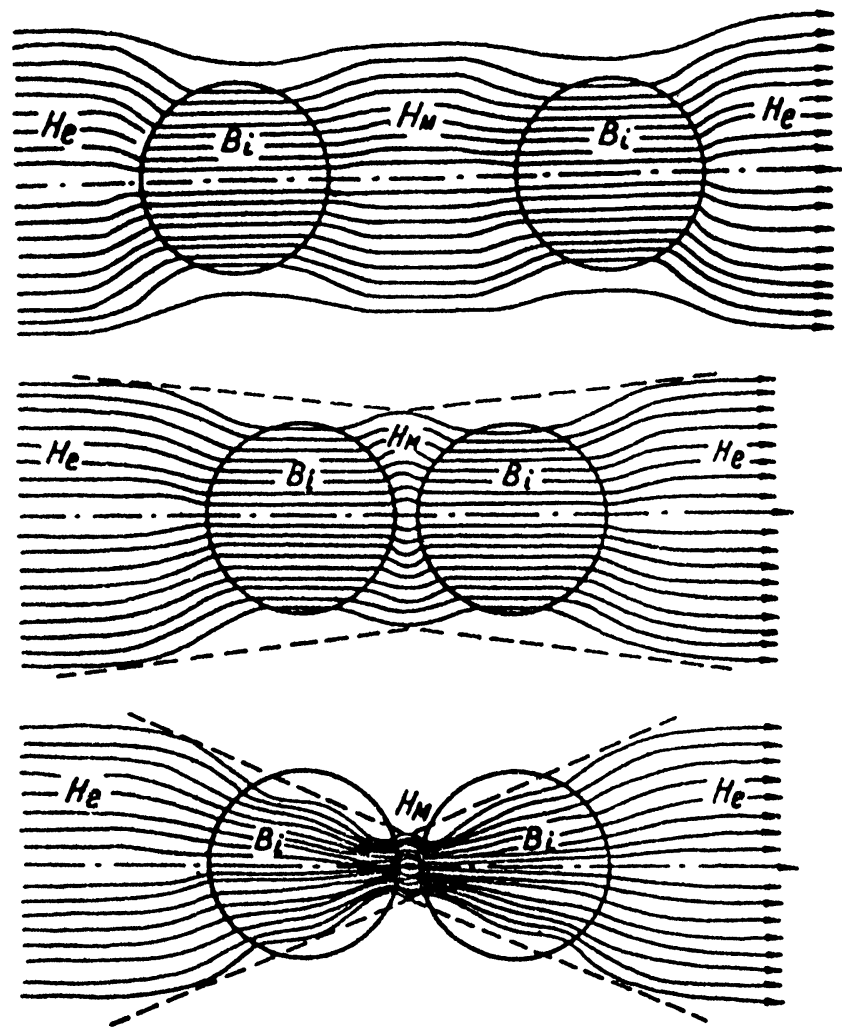

Fig. 6 Schematic diagram of formation of anticlinal gradients of the magnetic field strength which create ponderomotive forces of interaction when neighbouring particles and poles make contact

established experimentally, that the traction interaction between two particles becomes important when the distance between these particles decreases to five or fewer particle diameters. In the presence of sufficiently high level of magnetisation, flocculation even of uniformly magnetised paramagnetic or antiferromagnetic particles is possible. We have observed this phenomenon at high magnetic fields. 
Forces of the dipole interaction of magnetisable particles in a suspension can be estimated, for the flocculation volume, based on the Maxwell tensor, as tension $\sigma_{\mathbf{x}}$ on its boundary; e.g. along axis $x$ :

$$
\sigma_{\mathrm{x}}=\frac{\mu_{0}}{8 \pi} \mathrm{H}_{\mathrm{x}}^{2}\left[\frac{1+4 \pi \kappa}{1+4 \pi \mathrm{N} \kappa)^{2}}-1\right]
$$

where $\kappa$ is the magnetic susceptibility of the particles in a floc, $N$ is the demagnetisation factor of a floc, $\mu_{0}$ is the magnetic permeability of the medium and $H_{\mathrm{x}}$ is the magnetic field intensity along the axis $x$ of the floc. Tension along the remaining directions can be determined in a similar way.

The strength of the flocs formed in the magnetic field of contemporary magnetic separators with $\mathrm{H}>80 \mathrm{kA} / \mathrm{m}(1000 \mathrm{Oe})$ is determined by the magnetoadhesive forces. This was determined under various conditions (the effects of field intensity, shape of the flocs and concentration of the ferromagnetics were investigated). This force can as high as $0.6 \mathrm{~kg} / \mathrm{cm}^{2}[4]$.

In the process of high-gradient magnetic separation where the particles arrive into an immediate contact with a surface of the ferromagnetic poles, the dipole interaction of particles with the pole surfaces considerably facilitates the recovery, and particularly retention of magnetisable particles. for this case, it possible to make a more general, although a somewhat approximate estimate the magnetic force using the energy approach.

\section{THE ENERGY EVALUATION OF THE MAGNETIC FORCE}

It is well known that the general magnetic energy of a system "a pole-magnetic particle" is equal to:

$$
\mathrm{E}_{\mathrm{n}}=0.5 \int_{\mathrm{V}} \mathrm{B} \cdot \mathrm{H} \mathrm{dV}=\mathrm{const}
$$

This force change negligibly in the process of magnetic separation. Mechanical mass transfer work in all processes of magnetic separation is accomplished mainly 
as a result of a decrease of one of its components, namely the magnetostatic energy or of the energy of free poles:

$$
\mathrm{E}_{\mathrm{m}}=0.5 \int_{\mathrm{V}} \mathrm{J}^{2} \mathrm{~N} \mathrm{dV}
$$

where $N$ is the demagnetising factor. For a torroid, where there are no free poles, $\mathrm{N}=0$, and $\mathrm{E}_{\mathrm{m}}=0$.

When considering the limiting values of $E_{\mathrm{m}}$ for discrete elements of the system (approximate estimate), it is possible to determine the work $A$ performed in magnetic separation as a result of reduction of the energy of the free poles as a consequence of flocculation of particles and their attraction to the poles:

$$
\sum_{i=1}^{n} E_{m i}^{p}+\sum_{j=1}^{m} E_{m j}^{g}-\bar{E}_{m}=\Delta E_{m} \approx A
$$

where $\Sigma E_{m i}^{p}$ and $\Sigma E_{m j}^{g}$ are the total magnetostatic energies of the poles of a magnetic separator and of the flocs of the magnetic particles, respectively, and $E_{\mathrm{m}}$ is the reduced magnetostatic energy of the poles with a layer of particles attracted to the poles after separation.

The process of magnetic separation is of the continuous-cyclic character and the separation work is performed by the magnetic system in the process of attraction of particles of magnetic materials to the poles. The work is then returned to the magnetic system during the removal of the particles in the zone of removal of the magnetic fraction as a result of the work performed by the drum rotor, roll etc. causing the removal. This explains a long lifetime of drum magnetic separators with permanent magnets ( 2 to 3 years) without their additional remagnetisation.

If $\Delta \bar{E}_{\mathrm{m}} \approx A$, then the mean magnetic force acting in the working space in the direction of a pole is given by:

$$
\mathrm{F}_{\mathrm{m}}=-\frac{\partial \mathrm{E}_{\mathrm{m}}}{\partial \mathrm{x}}=-\frac{\Delta \mathrm{E}_{\mathrm{m}}}{\Delta \mathrm{x}} \simeq 0.5 \mathrm{~J}^{2} \mathrm{NS}
$$


where $S$ is the cross-sectional area of the contact surface.

If, for instance, the high-gradient medium in the volume $V$ of the working space are ferromagnetic spheres, surface area of which is:

$$
\mathrm{S}=\frac{4 \pi \mathrm{r}^{2} \mathrm{~V}}{(2 \mathrm{r})^{3}}-=\frac{\pi \mathrm{V}}{2 \mathrm{r}}
$$

where $r$ is the radius of the spheres, then

$$
\mathrm{F}_{\mathrm{rel}}=\frac{\mathrm{F}_{\mathrm{m}}}{\mathrm{F}_{\mathrm{g}}}=\frac{\mathrm{J}^{2} \mathrm{NV}}{16 \mathrm{r}} \approx \frac{\mathrm{C}_{0}}{\mathrm{r}}
$$

where $F_{\mathrm{g}}$ is the force of gravity and $C_{0}$ is the constant of the magnetic force which is a function of the magnetic properties of minerals. It can vary in a wide interval ranging from $10^{-2}$ to $10 \mathrm{~m}^{-1}$.

This is possible at high values of the magnetic induction in the working space of high-gradient magnetic separators, for which $\mathrm{J} \rightarrow \mathrm{J}_{\mathrm{s}}$ and $\mathrm{N} \approx$ const. for each type of matrix. In such a case the magnetic force depends only on the magnitude of the specific area of the poles, i.e. it is inversely proportional to the radius of the poles (17). In spite of approximations used to derive eq. (17), the equation is well confirmed by experimentally and can be used for engineering calculations.

The measurements were carried by a ponderomotive technique out in the gaps between the steel spheres of various sizes, using titanomagnetite particles. The results showed that the agreement between theory and experiment increases for small sizes (large curvature) where the dipole interaction between the particles and the poles is more dominant.

\section{CONCLUSIONS}

It follows from the previous discussion that the highest level of the force of the recovery is found in the immediate proximity of the surface the ferromagnetic bodies of the matrix. This fact must be taken into account when designing 
high-gradient magnetic separators, taking an advantage the film flow of the slurry over the matrix surface. Under these conditions the matrix surface is quickly covered with a monolayer of the magnetic fraction. If the magnetic fraction contains also strongly magnetic particles, a multilayer coverage of the matrix can be observed.

The amount of the magnetic fraction recovered per unit cross-sectional area of the matrix element, under the optimum conditions, is the fundamental characteristic and the main criterion in the selection of the type of the matrix.

The most interesting results in the application of high-gradient magnetic separation were obtained using the matrix of the second sort with the film layer. The beneficiation of the middlings, tailings and the slimes of rare and non-ferrous metals ( $\mathrm{Nb}, \mathrm{Ta}, \mathrm{W}, \mathrm{Mo}, \mathrm{Zn}$, Ti etc.) were successfully treated. Moreover, the concentration of sulphur in coals was reduced by a factor of two. The magnetic fraction contains $65 \%$ of sulphur and $70 \%$ of ash, as well as 60 to $70 \%$ of numerous toxic elements such as $\mathrm{As}, \mathrm{Pb}, \mathrm{V}, \mathrm{Cr}, \mathrm{Hg}, \mathrm{Co}, \mathrm{Mn}$ and others.

Magnetic and electrical methods allow to develop very efficient large-scale processes and separators. The reason is the presence of various magnetic and electric forces in the process of separation. These forces can achieve very high specific values and can considerably accelerate the mass transfer of the mineral particles in the separators. The application and deployment of these methods promote scientific and technical progress in the mining industry.

\section{REFERENCES}

[1] P.I. Zelenov et al.: Development of new processes for the beneficiation of magnetite-hematite ores at the Olenegorsk mining and beneficiation complex. Tenth International Mineral Processing Congress, London 1973

[2] E.M. Savitskiy et al: Separation of minerals using superconducting magnetic systems. Obogashch. briket. rud No.2(1973) (in Russian)

[3] I.E. Tamm: Fundamentals of the theory electricity. Nauka, Moscow, 1970 (in Russian) 
[4] V.I. Karmazin and V.V. Karmazin: Magnetic methods of beneficiation. Nedra, Moscow 1984 (in Russian)

[5] V.V. Karmazin and V.I. Karmazin: Magnetic and electrical methods of beneficiation. Nedra, Moscow 1988 (in Russian)

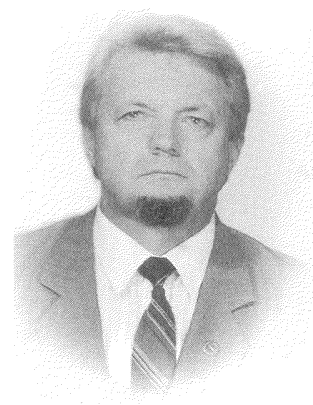

Victor Vitalyevich Karmazin was born in 1936 and graduated from the Krivoy Rog Ore Mining Institute in 1958, in the field of ore dressing. He worked as a chief engineer at the Central Beneficiation Plant of the Nicopol-Margantes trust. In 1964 he obtained his M.Sc. degree from the Mining Institute, Academy of Sciences of the USSR in Moscow. From 1971 to 1983 he was a lecturer at the Moscow Mining Institute where he also obtained his D.Sc. degree. From 1983 to 1991 he was the rector of the North-Caucasus Mining and Metallurgical Institute and the head of the Ore-dressing Department. Since 1991 he has been the professor at the Moscow State Mining University. Professor Karmazin is the author of seven monographs, three textbooks and more than 150 papers and patents in the field of magnetic and electrical methods of beneficiation or ores.

Keywords: magnetic separation, electrical separation, ponderomotive force, magnetoadhesive force, superconducting separation, high-gradient magnetic separation 